\title{
Production of plasmid-encoding NDM-1 in clinical Raoultella ornithinolytica and Leclercia adecarboxylata from China
}

\section{OPEN ACCESS}

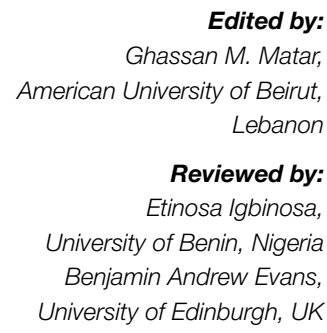

Edited by:

Ghassan M. Matar,

American University of Beirut,

Lebanon

Reviewed by:

Etinosa Igbinosa,

University of Benin, Nigeria

Benjamin Andrew Evans,

University of Edinburgh, UK

${ }^{*}$ Correspondence:

Peiyuan Xia

Department of Pharmacy, Southwest Hospital, Third Military Medical University, No. 30, Gaotanyan, Shapingba District, Chongqing 400038, China

py_xia2013@163.com;

Dongsheng Zhou,

State Key Laboratory of Pathogen and Biosecurity, Beijing Institute of Microbiology and Epidemiology, No.

20, Dongdajie, Fengtai, Beijing 100853, China dongshengzhou1977@gmail.com

Specialty section:

This article was submitted to Antimicrobials, Resistance and Chemotherapy, a section of the journal Frontiers in Microbiology

Received: 16 February 2015 Accepted: 27 April 2015

Published: 21 May 2015

Citation:

Sun F, Yin Z, Feng J, Qiu Y, Zhang D, Luo $W$, Yang $H$, Yang $W$, Wang J, Chen W, Xia P and Zhou D (2015)

Production of plasmid-encoding NDM-1 in clinical Raoultella ornithinolytica and Leclercia adecarboxylata from China.

Front. Microbiol. 6:458

doi: 10.3389/fmicb.2015.00458

\author{
Fengjun Sun ${ }^{1}$, Zhe Yin ${ }^{2}$, Jiao Feng ${ }^{2,3}$, Yefeng Qiu ${ }^{3}$, Defu Zhang ${ }^{2}$, Wenbo Luo ${ }^{1,2}$, \\ Huiying Yang ${ }^{2}$, Wenhui Yang ${ }^{2}$, Jie Wang ${ }^{2}$, Weijun Chen ${ }^{4}$, Peiyuan Xia ${ }^{1 *}$ and \\ Dongsheng Zhou ${ }^{2 *}$ \\ ${ }^{1}$ Department of Pharmacy, Southwest Hospital, The Third Military Medical University, Chongqing, China, ${ }^{2}$ State Key \\ Laboratory of Pathogen and Biosecurity, Beijing Institute of Microbiology and Epidemiology, Beijing, China, ${ }^{3}$ Laboratory \\ Animal Center, Academy of Military Medical Sciences, Beijing, China, ${ }^{4}$ Beijing Institute of Genomics, Chinese Academy of \\ Sciences, Beijing, China
}

Raoultella ornithinolytica YNKP001 and Leclercia adecarboxylata P10164, which harbor conjugative plasmids pYNKP001-NDM and pP10164-NDM, respectively, were isolated from two different Chinese patients, and their complete nucleotide sequences were determined. Production of NDM-1 enzyme by these plasmids accounts for the carbapenem resistance of these two strains. This is the first report of bla NDM in $L$. adecarboxylata and third report of this gene in $R$. ornithinolytica. pYNKP001-NDM is very similar to the IncN2 NDM-1-encoding plasmids pTR3, pNDM-ECS01, and

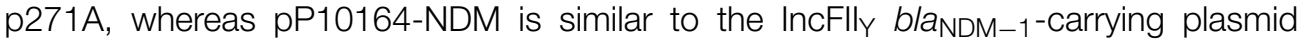
pKOX_NDM1. The bla NDM-1 genes of pYNKP001-NDM and pP10164-NDM are embedded in Tn125-like elements, which represent two distinct truncated versions of the NDM-1-encoding Tn125 prototype observed in pNDM-BJ01. Flanking of these two Tn125-like elements by miniature inverted repeat element (MITE) or its remnant indicates that MITE facilitates transposition and mobilization of bla $\mathrm{NDM}_{-1}$ gene contexts.

Keywords: Raoultella ornithinolytica, Leclercia adecarboxylata, NDM-1, plasmid, carbapenem resistance

\section{Introduction}

Raoultella ornithinolytica is widely found in aquatic environments, insects and fishes. $R$. ornithinolytica is able to convert histidine to histamine (scombroid toxin) and is thus known to cause fish poisoning. Symptoms primarily manifest with facial flushing, dizziness, vomiting, diarrhea, dyspnea, headache, urticarial, and generalized pruritus and commonly subside in a few hours (Kanki et al., 2002). Infections by R. ornithinolytica are exceedingly rare in humans and have been reported as bloodstream, urinary tract and soft tissue infections in adults and as fatal neonatal infections. Most adult cases are linked with underlying diseases, especially malignancies (Morais et al., 2009; Mau and Ross, 2010; Solak et al., 2011; Hadano et al., 2012; Haruki et al., 2014; Chun et al., 2015).

Raoultella ornithinolytica produces at least two different chromosomally encoded class A $\beta$-lactamases. Accordingly, $R$. ornithinolytica is resistant to ampicillin but commonly remains susceptible to cefotaxime and imipenem (Walckenaer et al., 2004). Notably, carbapenem-resistant 
$R$. ornithinolytica has been reported due to the production of plasmid-encoding carbapenemase KPC-3 (Castanheira et al., 2009) or NDM-1 (Khajuria et al., 2013; Zhou et al., 2014).

Leclercia adecarboxylata is a ubiquitous organism that is rarely clinically isolated in humans. However, L. adecarboxylata has been recognized as an opportunistic pathogen in immunocompromised patients suffering from primary diseases and often depends on co-flora to cause polymicrobial infection (Shin et al., 2012; De Mauri et al., 2013; Garcia-Fulgueiras et al., 2014). L. adecarboxylata is isolated from various clinical specimens (e.g., blood, feces, sputum, urine, and wound pus) and causes bacteremia, endocarditis, sepsis, peritonitis, cellulitis, endocarditis, and cholecystitis (Shin et al., 2012; De Mauri et al., 2013; Garcia-Fulgueiras et al., 2014). L. adecarboxylatainduced monomicrobial infections (e.g., wound infection, pharyngeal abscess, and bacteremia) have also been reported in immunocompetent patients (Hess et al., 2008; Bali et al., 2013; Michael et al., 2013; Garcia-Fulgueiras et al., 2014; Keren et al., 2014).

Leclercia adecarboxylata is generally susceptible to commonly used antibiotics, but there are a few reports of L. adecarboxylata harboring different antibiotic resistance mechanisms. Cephalosporin- and carbapenem-resistant strains of $L$. adecarboxylata have been identified due to the production of extended-spectrum $\beta$-lactamase (ESBL) SHV-12 (Mazzariol et al., 2003) and carbapenemase KPC-2 (Geffen et al., 2013) or VIM-1 (Papagiannitsis et al., 2013), respectively. Notably, clinical isolates of multidrug-resistant $L$. adecarboxylata have been known to harbor multiple antibiotic resistance genes that are captured by class 1 integrons (Yao et al., 2011; Shin et al., 2012; Garcia-Fulgueiras et al., 2014).

NDM is an Ambler class B metallo- $\beta$-lactamase that confers resistance to nearly all $\beta$-lactam antibiotics, including carbapenems, and $b l a_{\mathrm{NDM}}$ genes have been identified in a large array of Enterobacteriaceae species (Nordmann et al., 2011; Johnson and Woodford, 2013; Dortet et al., 2014). Both $R$. ornithinolytica and $L$. adecarboxylata are members of Enterobacteriaceae. In this study, we analyzed complete nucleotide sequences of two different NDM-1-encoding plasmids, pYNKP001-NDM, and pP10164-NDM, recovered from R. ornithinolytica YNKP001 and L. adecarboxylata P10164, respectively, of clinical origin in China.

\section{Materials and Methods}

\section{Bacterial Strains and Identification}

The use of human specimens and all related experimental protocols were approved by the Committee on Human Research of indicated institutions and carried out in accordance with the approved guidelines. Informed consent was obtained from the indicated patients. All bacterial strains were subjected to species identification by BioMérieux VITEK 2, Bruker MALDI Biotyper, and 16S rRNA gene sequencing. For $16 \mathrm{~S}$ rRNA gene sequence determination, nearly the complete coding region of $16 \mathrm{~S}$ rRNA gene was amplified by PCR with the universal primers 27f (AGAGTTTGATCCTGGCTCAG) and 1492r (TACCTTGTTACGACTT) (Frank et al., 2008). The major carbapenemase and ESBL genes (Table S1) were subjected to PCR detection. All PCR amplicons were sequenced on an ABI 3730 Sequencer with the same primers for PCR.

\section{Plasmid Conjugal Transfer}

Plasmid conjugal transfer experiments were carried out with Escherichia coli EC600 (rifampin-resistant) or TB1 (streptomycin-resistant) used as recipient and the $b l a_{\mathrm{NDM}^{-}}$ positive strain YNKP001 or P10164 as donor. Then, $3 \mathrm{ml}$ of overnight culture of each of donor and recipient bacteria were mixed together, harvested and resuspended in $80 \mu \mathrm{l}$ of brain heart infusion (BHI) broth (BD Biosciences). The mixture was spotted on a $1-\mathrm{cm}^{2}$ filter membrane placed on BHI agar (BD Biosciences) plates. The plates were incubated for mating at $37^{\circ} \mathrm{C}$ for $12-18 \mathrm{~h}$. Bacteria were washed from filter membrane and spotted on Muller-Hinton ( $\mathrm{MH}$ ) agar (BD Biosciences) plates containing $1000 \mu \mathrm{g} / \mathrm{ml}$ rifampin or $250 \mu \mathrm{g} / \mathrm{ml}$ streptomycin and $200 \mu \mathrm{g} / \mathrm{ml}$ ampicillin for selection of $b l a_{\mathrm{NDM}}$-positive $E$. coli transconjugants.

\section{Detection of Carbapenemase Activity}

Activity of class A/B/D carbapenemases was determined via a CarbaNP test (Dortet et al., 2012) with modifications. Overnight bacterial cell culture in $\mathrm{MH}$ broth was diluted 1:100 into $3 \mathrm{ml}$ of fresh $\mathrm{MH}$ broth, and bacteria were allowed to grow at $37^{\circ} \mathrm{C}$ with shaking at $200 \mathrm{rpm}$ to reach an $\mathrm{OD}_{600}$ of 1.0-1.4. If required, ampicillin was used at $200 \mu \mathrm{g} / \mathrm{ml}$. Bacterial cells were harvested from $2 \mathrm{ml}$ of the above culture and washed twice with $20 \mathrm{mM}$ Tris- $\mathrm{HCl}(\mathrm{pH} 7.8)$. Cell pellets were resuspended in $500 \mu \mathrm{l}$ of $20 \mathrm{mM}$ Tris- $\mathrm{HCl}(\mathrm{pH} \mathrm{7.8)}$ and lysed by sonication, followed by centrifugation at $10000 \times \mathrm{g}$ at $4^{\circ} \mathrm{C}$ for $5 \mathrm{~min}$. Then, $50 \mu \mathrm{l}$ of the supernatant (enzymatic bacterial suspension) was mixed with $50 \mu \mathrm{l}$ of substrate $\mathrm{I}-\mathrm{V}$, followed by incubation at $37^{\circ} \mathrm{C}$ for a maximum of $2 \mathrm{~h}$. Substrate I: $0.054 \%$ red phenol plus $0.1 \mathrm{mM} \mathrm{ZnSO}_{4}$ (pH 7.8). Substrate II: $0.054 \%$ red phenol plus $0.1 \mathrm{mM} \mathrm{ZnSO}_{4}(\mathrm{pH} 7.8)$ and $0.6 \mathrm{mg} / \mu \mathrm{l}$ imipenem. Substrate III: $0.054 \%$ red phenol plus $0.1 \mathrm{mM} \mathrm{ZnSO}_{4}(\mathrm{pH} 7.8), 0.6 \mathrm{mg} / \mu \mathrm{lmg}$ imipenem, and $0.8 \mathrm{mg} / \mu \mathrm{l}$ tazobactam. Substrate IV: $0.054 \%$ red phenol plus $0.1 \mathrm{mM} \mathrm{ZnSO}_{4}(\mathrm{pH} 7.8), 0.6 \mathrm{mg} / \mu \mathrm{lmg}$ imipenem, and $3 \mathrm{mM}$ EDTA (pH 7.8). Substrate V: $0.054 \%$ red phenol plus $0.1 \mathrm{mM} \mathrm{ZnSO}_{4}(\mathrm{pH} 7.8), 0.6 \mathrm{mg} / \mu \mathrm{lmg}$ imipenem, $0.8 \mathrm{mg} / \mu \mathrm{l}$ tazobactam, and 3 mM EDTA (pH 7.8).

\section{Determination of Minimum Inhibitory Concentration (MIC)}

The MIC values of the indicated bacterial strains were tested by VITEK 2 according to the manufacturer's instructions, and antimicrobial susceptibility was assessed by the Clinical and Laboratory Standards Institute (CLSI) standards.

\section{Determination of Plasmid DNA Sequences}

The chromosome DNA-free plasmid DNA was isolated from the cell cultures of indicated $E$. coli transconjugant using a Qiagen large construct kit and then sequenced using the wholegenome shotgun strategy in combination with Illumina HiSeq 2500 sequencing technology. The contigs were assembled with Velvet, and the gaps were filled through combinatorial PCR and 
Sanger sequencing with an ABI 3730 Sequencer. The genes were predicted with GeneMarkS and further annotated by BLASTP against UniPort and NR databases.

\section{Nucleotide Sequence Accession Numbers}

The complete sequences of plasmids pP10164-NDM and pYNKP001-NDM were submitted to GenBank under accession numbers KP900016 and KP900017, respectively.

\section{Results}

\section{Characterization of $R$. ornithinolytica YNKP001 and $L$. adecarboxylata P10164}

Raoultella ornithinolytica YNKP001 was recovered in November 2010 from the blood specimens of a 4-year-old child with acute encephalitis, bronchitis and tympanitis from a hospital in Kunming City in China. L. adecarboxylata P10164 was isolated in August 2012 from the sputum specimens of a 43year-old male with pneumonia admitted to a teaching hospital in Chongqing City in China. Both patients received empirical intravenous administration with ceftazidime for at least 1 week, but their symptoms did not improve. Subsequent antimicrobial susceptibility tests indicated that both strains were resistant to multiple $\beta$-lactams, including imipenem, and meropenem, but remained susceptible to fluoroquinolones. The patients then received intravenous administration with moxifloxacin and were cured and discharged approximately 10 days after initiating antimicrobial treatment.

Plasmids pP10164-NDM and pYNKP001-NDM could be readily transferred from $\mathrm{P} 10164$ and YNKP001 into E. coli EC600 and TB1, respectively, generating two corresponding E. coli transconjugants: P10164-NDM-EC600 and YNKP001NDM-TB1. PCR detection of the major ESBL and carbapenemase genes (Table S1) indicated that P10164 harbored the bla $a_{\mathrm{NDM}}$, bla $a_{\mathrm{CTX}-\mathrm{M}-1}$ group, bla $a_{\mathrm{TEM}}$, and bla $a_{\mathrm{OXA}-1}$ group genes, whereas YNKP001, P10164-NDM-EC600 and YNKP001NDM-TB1 contained only bla $a_{\mathrm{NDM}}$, as confirmed by PCR amplicon sequencing. Class $\mathrm{B}$ carbapenemase activity was detected in P10164, YNKP001, P10164-NDM-EC600 and YNKP001-NDM-TB1 (Figure S1).

P10164 was resistant to all of the tested drugs, including penicillin, $\beta$-lactam inhibitor, cephalosporin, carbapenem, monobactam, fluoroquinolone, furane, and aminoglycoside (Table 1). YNKP001, P10164-NDM-EC600, and YNKP001NDM-TB1 were resistant to penicillins, $\beta$-lactam inhibitors, cephalosporins, and carbapenems but remained susceptible to fluoroquinolones, aminoglycosides, aztreonam, and macrodantin (Table 1).

\section{Comparative Genomics of pYNKP001-NDM}

As revealed by high-throughput sequencing with a mean coverage fold of 143, plasmid pYNKP001-NDM was 41,190 bp in size with a mean GC content of $50.8 \%$ and contained 54 open reading frames (ORFs) (Figure 1A). The replication module present on pYNKP001-NDM belonged to the IncN2 incompatibility group (Poirel et al., 2011). This plasmid was mostly similar to another NDM-encoding IncN2 plasmid, pTR3
(Chen et al., 2012), with genetic differences in only three single nucleotide polymorphisms (SNPs).

Further linear genomic comparison (Figure 2A) was performed with all four NDM-encoding IncN2 plasmids, namely, pYNKP001-NDM, pTR3 (Chen et al., 2012), pNDMECS01 (Netikul et al., 2014), and p271A (Poirel et al., 2011). The former three plasmids essentially had the same genomic organization. The genome of each plasmid could be divided into the backbone and accessory module. The backbone was composed of genes responsible for plasmid replication (repA), stability ( $s t b A B C, s s b, k o r B, k l c A$, etc.) and conjugal transfer (tra). p271A lacked a $5.2 \mathrm{~Kb}$ region found in pYNKP001-NDM, pTR3, and pNDM-ECS01. This region corresponded to the CUP (conserved upstream repeat)-controlled regulon commonly found in the IncN1 and IncN2 plasmids, and the loss of this region might be due to recombination between CUPs (Partridge et al., 2012).

The four plasmids shared a single conserved accessory module, which was sequentially organized as an intact miniature

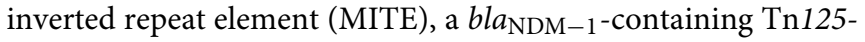
like element, a MITE remnant, an intact ISSen4 element with $26 \mathrm{bp}$ inverted repeats (IRs) at both sides, a truncated aphA6 (aminoglycoside resistance) gene, and an intact Tn5403 element with 39 bp IRs at both ends (Figure 3A). The Tn125 prototype was sequentially organized as ISAba125, bla $a_{\mathrm{NDM}-1}$, ble $_{\mathrm{MBL}}$ (bleomycin resistance), $\triangle \operatorname{trpF}, d s b C$, cutA, $\triangle$ groES, groEL, ISCR27, and ISAba125; Tn125 was a typical composite transposon (Tn) containing two flanking copies of ISAba125 (each with $26 \mathrm{bp}$ IRs at both ends); Tn 125 could be inserted into a site downstream of aphA6, leaving 3 bp direct repeats (DRs) at both ends, as observed in pNDM-BJ01 (Hu et al., 2012; Poirel et al., 2012) (Figure 3A). Compared with the Tn125 prototype, the Tn125-like element in pYNKP001-NDM lacked the entire fragment of $d s b C, c u t A, \triangle g r o E S$, groEL, ISCR27, and ISAba125. In addition, the upstream copy of ISAba125 was a truncated version lacking the left IR and was interrupted by ISEc33 (with $17 \mathrm{bp}$ IRs at both ends) (Figure 3A).

The flanking of a genetic context (e.g., class 1 integron) by two identical MITEs has been recently characterized as a mechanism for mobilizing antimicrobial resistance determinants via MITE-mediated transposition or homologous recombination (Poirel et al., 2009; Domingues et al., 2013; Zong, 2014). Notably, the Tn125-like element in pYNKP001-NDM was embedded between an intact $257 \mathrm{bp}$ MITE and a $55 \mathrm{bp}$ MITE remnant (Figure 3A). The intact MITE contained $39 \mathrm{bp}$ IRs and could potentially form a long stem-loop RNA structure (Delihas, 2011), whereas the downstream MITE remnant corresponded to the $3^{\prime}$-terminal $55 \mathrm{bp}$ fragment (including the right IR) of the intact MITE.

\section{Comparative Genomics of pP10164-NDM}

Plasmid pP10164-NDM was fully sequenced, with a mean coverage fold of 248 . Moreover, it was 99,276 bp in size, with a mean GC content of 55\%, and contained 101 ORFs (Figure 1B). pP10164-NDM was assigned to the IncFII group encoding two different replication proteins $\operatorname{IncFII}_{\mathrm{Y}}($ repA) and IncFIB (repB) (Villa et al., 2010). Comparative genomics 
TABLE 1 | MIC values and antimicrobial susceptibility.

\begin{tabular}{|c|c|c|c|c|c|c|c|}
\hline \multirow[t]{2}{*}{ Category } & \multirow[t]{2}{*}{ Antibiotics } & \multicolumn{6}{|c|}{ MIC $(\mu \mathrm{g} / \mathrm{ml}) /$ antimicrobial susceptibility } \\
\hline & & P10164 & P10164-NDM-EC600 & EC600 & YNKP001 & YNKP001-NDM -TB1 & TB1 \\
\hline \multirow[t]{4}{*}{ Penicillins } & Ampicillin & $\geq 32 / R$ & $\geq 32 / R$ & $16 / 1$ & $\geq 32 / R$ & $\geq 32 / R$ & $16 / l$ \\
\hline & Ampicillin/sulbactam & $\geq 32 / R$ & $\geq 32 / R$ & $8 / \mathrm{S}$ & $\geq 32 / R$ & $\geq 32 / R$ & $8 / \mathrm{S}$ \\
\hline & Piperacillin & $\geq 128 / R$ & $\geq 128 / R$ & $\leq 4 / \mathrm{S}$ & $\geq 128 / R$ & $\geq 128 / R$ & $\leq 4 / S$ \\
\hline & Piperacillin/tazobactam & $\geq 128 / R$ & $\geq 128 / R$ & $\leq 4 / \mathrm{S}$ & $\geq 128 / R$ & $\geq 128 / R$ & $\leq 4 / S$ \\
\hline \multirow[t]{6}{*}{ Cephalosporins } & Cefazolin & $\geq 64 / R$ & $\geq 64 / R$ & $\leq 4 / \mathrm{S}$ & $\geq 64 / R$ & $\geq 64 / R$ & $\leq 4 / S$ \\
\hline & Cefuroxime sodium & $\geq 64 / R$ & $\geq 64 / R$ & $16 / 1$ & $\geq 64 / R$ & $\geq 64 / R$ & $16 / S$ \\
\hline & Cefuroxime axetil & $\geq 64 / \mathrm{R}$ & $\geq 64 / R$ & $16 / l$ & $\geq 64 / R$ & $\geq 64 / R$ & $16 / S$ \\
\hline & Cefotetan & $\geq 64 / R$ & $\geq 64 / R$ & $\leq 4 / S$ & $\geq 64 / R$ & $\geq 64 / R$ & $\leq 4 / S$ \\
\hline & Ceftriaxone & $\geq 64 / R$ & $\geq 64 / R$ & $\leq 1 / \mathrm{S}$ & $\geq 64 / R$ & $\geq 64 / R$ & $\leq 1 / \mathrm{S}$ \\
\hline & Ceftazidime & $\geq 64 / R$ & $\geq 64 / R$ & $\leq 1 / \mathrm{S}$ & $\geq 64 / R$ & $\geq 64 / R$ & $\leq 1 / \mathrm{S}$ \\
\hline \multirow[t]{2}{*}{ Carbapenems } & Imipenem & $\geq 16 / R$ & $\geq 16 / R$ & $\leq 1 / \mathrm{S}$ & $\geq 16 / R$ & $\geq 16 / R$ & $\leq 1 / \mathrm{S}$ \\
\hline & Meropenem & $8 / \mathrm{R}$ & 4/R & $\leq 0.25 / \mathrm{S}$ & $\geq 16 / R$ & $\geq 16 / R$ & $\leq 0.25 / \mathrm{S}$ \\
\hline Monobactams & Aztreonam & $16 / R$ & $\leq 1 / S$ & $\leq 1 / \mathrm{S}$ & $\leq 1 / S$ & $\leq 1 / \mathrm{S}$ & $\leq 1 / \mathrm{S}$ \\
\hline \multirow[t]{2}{*}{ Fluoroquinolones } & Ciprofloxacin & $\geq 4 / R$ & $\leq 0.25 / \mathrm{S}$ & $\leq 0.25 / \mathrm{S}$ & $\leq 0.25 / \mathrm{S}$ & $\leq 0.25 / \mathrm{S}$ & $\leq 0.25 / \mathrm{S}$ \\
\hline & Levofloxacin & $\geq 8 / R$ & $0.5 / \mathrm{S}$ & $0.5 / \mathrm{S}$ & $0.5 / \mathrm{S}$ & $\leq 0.25 / \mathrm{S}$ & $\leq 0.25 / \mathrm{S}$ \\
\hline Furanes & Macrodantin & $\geq 512 / R$ & $\leq 16 / \mathrm{S}$ & $\leq 16 / S$ & $32 / S$ & $\leq 16 / S$ & $\leq 16 / S$ \\
\hline \multirow[t]{3}{*}{ Aminoglycosides } & Amikacin & $\geq 64 / R$ & $\leq 2 / \mathrm{S}$ & $\leq 2 / S$ & $\leq 2 / S$ & $\leq 2 / S$ & $\leq 2 / S$ \\
\hline & Gentamicin & $\geq 16 / R$ & $\leq 1 / \mathrm{S}$ & $\leq 1 / \mathrm{S}$ & $\leq 1 / \mathrm{S}$ & $\leq 1 / \mathrm{S}$ & $\leq 1 / \mathrm{S}$ \\
\hline & Tobramycin & $\geq 16 / R$ & $\leq 1 / \mathrm{S}$ & $\leq 1 / \mathrm{S}$ & $\leq 1 / \mathrm{S}$ & $\leq 1 / \mathrm{S}$ & $\leq 1 / \mathrm{S}$ \\
\hline
\end{tabular}

analysis was performed with the only two characterized NDMencoding IncFIIY plasmids: pP10164-NDM and pKOX_NDM1 (Huang et al., 2013) (Figure 2B). pP10164-NDM differed from pKOX_NDM1 in 214 SNPs, 11 single-nucleotide indels, and three large deletions (11285 bp, $180 \mathrm{bp}$, and $45 \mathrm{bp}$, respectively). pP10164-NDM and pKOX_NDM1 had very similar backbones composed of genes responsible for plasmid replication (repA and $r e p B$ ), stability ( $p m a A B, p s i A B, k l c A, y u b$, etc.) and conjugal transfer (tra); the $45 \mathrm{bp}$ deletion (nucleotide position 102082 to 102126 in pKOX_NDM1; located within the traD gene) was the only observed structural difference between the backbones of pP10164-NDM and pKOX_NDM1 (Figure 2B).

pP10164-NDM contained a single accessory module that was $38,098 \mathrm{bp}$ in size, in which the $11285 \mathrm{bp}$ and $180 \mathrm{bp}$ deletions (nucleotide positions 10620-21904 and 37157-37336 in pKOX_NDM, respectively) compared with the counterpart of pKOX_NDM1 were located (Figure 3B). The accessory module of pKOX_NDM1 harbored three copies of $256 \mathrm{bp}$ MITEs highly similar to the above-mentioned $257 \mathrm{bp}$ MITE, constituting a linear structure organized as the $11285 \mathrm{bp}$ region (MITE plus $11029 \mathrm{bp}$ region), MITE, Tn125-like element, and MITE (Figure 3B). Homologous recombination mediated by the first two copies of MITE appeared to lead to the insertion of the 11285 bp region into pKOX_NDM1 relative to pP10164NDM (Figure 3B). In addition to the first copy of MITE, the $11285 \mathrm{bp}$ region still contained $r m t C$ (16S rRNA methylase for aminoglycoside resistance), ISCR14 and an uncharacterized Tn (tniB, $\Delta$ tniA, and tniA) (Figure 3B).

The $b l a_{\mathrm{NDM}-1}$-harboring Tn125-like element was flanked by the second and third copies of MITE, indicating a similar mechanism of MITE-mediated insertion to the Tn125-like element (Figure 3B). Compared with the Tn125 prototype in pNDM-BJ01, the Tn125-like element in pP10164-NDM (Figure 3B) exhibited absence of a downstream copy of ISAba125, as well as truncation of ISCR27 and an upstream copy of ISAba125.

In addition, three potential composite Tns, with the characteristic of being flanked by two separate copies of IS5 or IS1, were identified within the accessory module of pP10164NDM or pKOX_NDM1, but none of the flanking DRs as target sites could be found.

\section{Discussion}

Horizontal transfer of plasmid-borne $b l a_{\mathrm{NDM}}$ genes enabled NDM enzymes to be rapidly spread in Enterobacteriaceae, less frequently in Acinetobacter, and rarely in Pseudomonas (Nordmann et al., 2011; Johnson and Woodford, 2013; Dortet et al., 2014). Klebsiella pneumoniae and E. coli are the most commonly described bla $a_{\mathrm{NDM}}$-carrying Enterobacteriaceae species, and $b l a_{\mathrm{NDM}}$ genes have been described in many other enterobacterial species, such as K. oxytoca, K. ozaenae, 

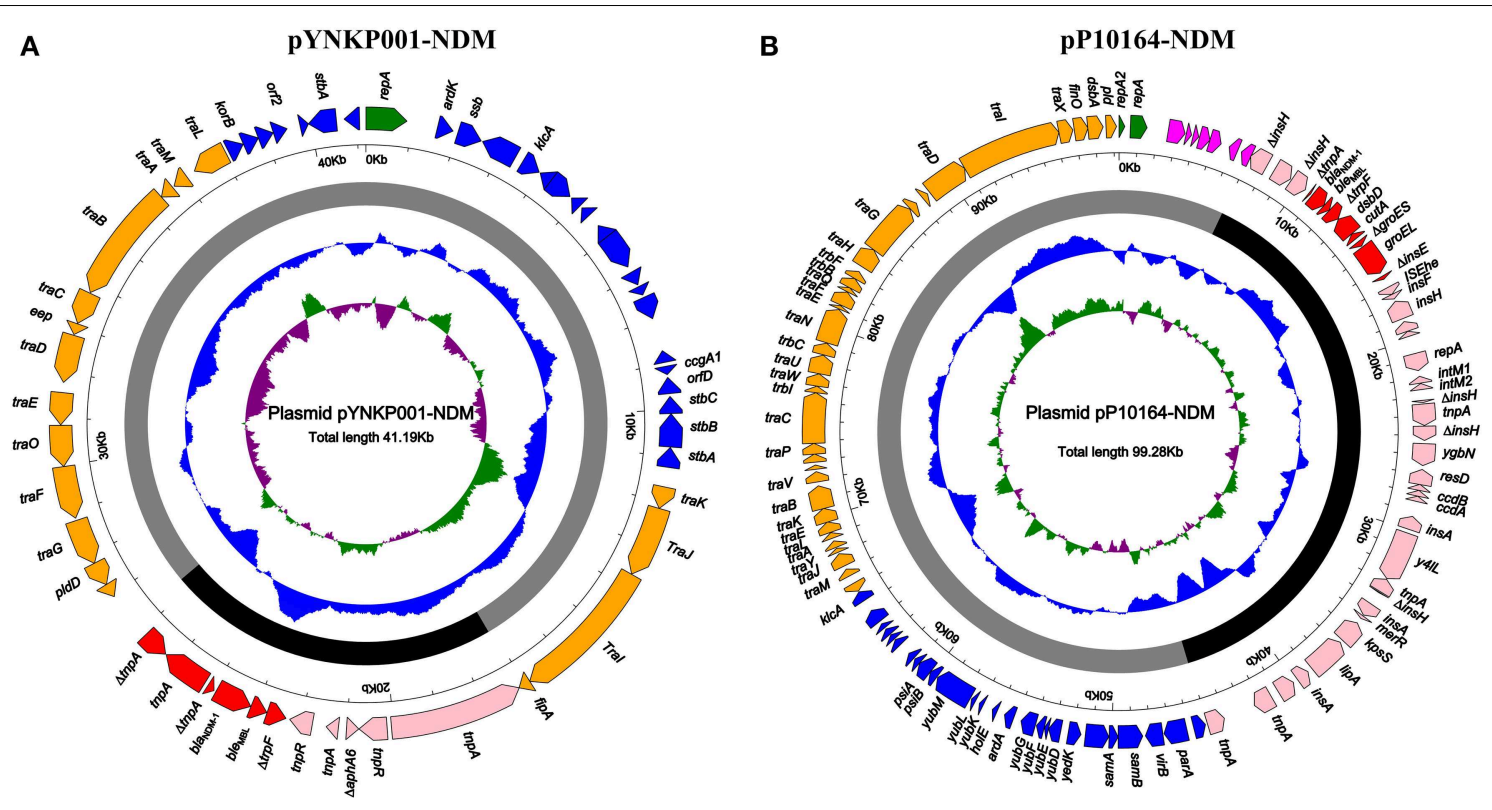

\begin{tabular}{|c|c|c|}
\hline Backbone $\square$ & $\begin{array}{l}\text { Plasmid replication } \\
\text { Plasmid stability } \\
\text { Conjugal transfer } \\
\text { Others }\end{array}$ & Accessory modules $\square\left[\begin{array}{l}\square \text { bla } a_{\mathrm{NDM}-1} \text {-containing Tn125-like element } \\
\square \text { Others }\end{array}\right.$ \\
\hline
\end{tabular}

FIGURE 1 | Schematic maps of plasmids pYNKP001-NDM (A) and pP10164-NDM (B). Genes are denoted by arrows and colored based on gene function classification. The innermost circle presents GC-Skew [(G -
$C) /(G+C)]$ with a window size of $500 \mathrm{bp}$ and step size of $20 \mathrm{bp}$. The blue circle presents GC content. Backbone and accessory module regions are also shown.

\section{A pYNKP001-NDM

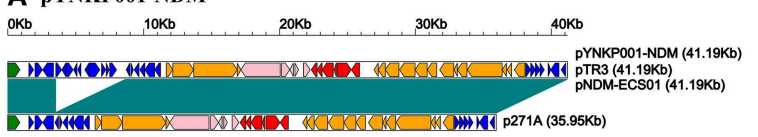

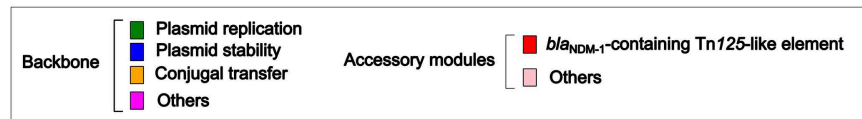 \\ B pP10164-NDM

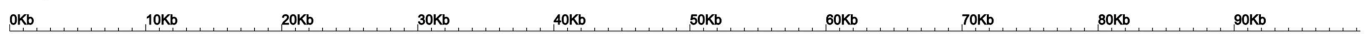

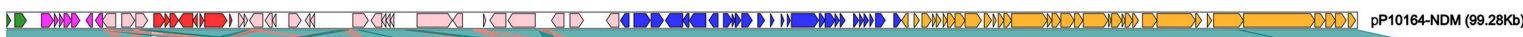

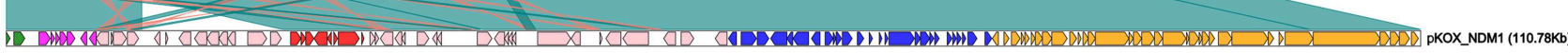

FIGURE 2 | Linear comparison of YNKP001-NDM (A) or pP10164-NDM (B) with its closely related plasmid. Genes are denoted by arrows and colored based on gene function classification. Dark green shading denotes shared regions of homology ( $>98 \%$ nucleotide similarity).

Citrobacter freundii, Enterobacter aerogenes, Enterobacter cloacae, Proteus mirabilis, Morganella morganii, Providencia spp., Serratia marcescens, and Salmonella enterica (Ou et al., 2014; Biedenbach et al., 2015). These NDM-producing bacteria have been shown to cause hospital- and community-acquired infections (Ou et al., 2014; Biedenbach et al., 2015). bla $a_{\mathrm{NDM}-1^{-}}$ positive bacteria are frequently found in environmental settings, indicating environmental origins of bla $a_{\mathrm{NDM}}$ genes in human pathogens, whereas use of antimicrobial drugs in an indiscriminate manner in some countries makes bla $a_{\mathrm{NDM}-1^{-}}$ positive bacteria spread easily and pose a serious public health threat (Walsh et al., 2011; Isozumi et al., 2012).

This is the first report of bla $a_{\mathrm{NDM}}$ in L. adecarboxylata. The clinical $L$. adecarboxylata isolate characterized herein harbors a conjugative IncFIIY plasmid pP10164-NDM that encodes the NDM-1 enzyme. pP10164-NDM is highly similar to pKOX_NDM1, which is recovered from a nosocomial $K$. oxytoca strain from a patient following medical transfer from a hospital in Jiangxi to Taiwan, China (Huang et al., 2013). There are only two reports of bla $a_{\mathrm{NDM}}$ in $R$. ornithinolytica, one from India (Khajuria et al., 2013) and the other from China (Zhou et al., 2014), both of which were confined to surveillance of $b l a_{\mathrm{NDM}}$ genes. This work presents extended evidence that the production of NDM-1 by a conjugative IncN2 plasmid pYNKP001-NDM accounts for the carbapenem resistance of a clinical $R$. ornithinolytica isolate from China. pYNKP001-NDM is very similar to pTR3 (identified in $K$. pneumoniae from a Chinese patient) (Chen et al., 2012), pNDMECS01 (in E. coli from a Thai patient) (Netikul et al., 2014) and 


\section{A pYNKP001-NDM}
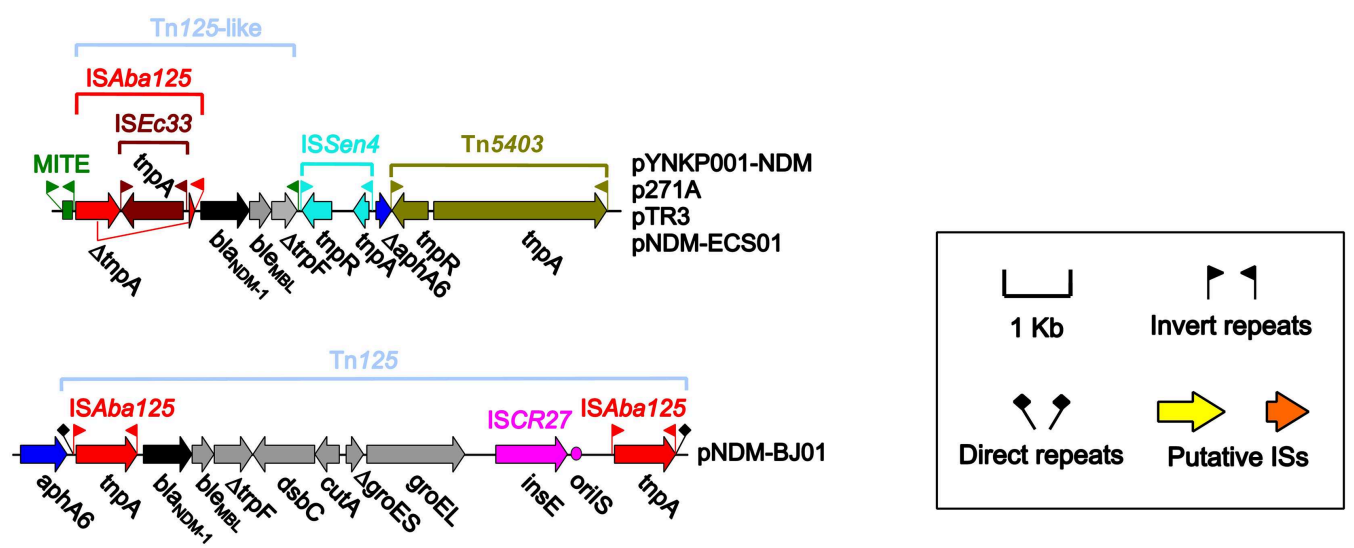

B $\quad$ PP10164-NDM

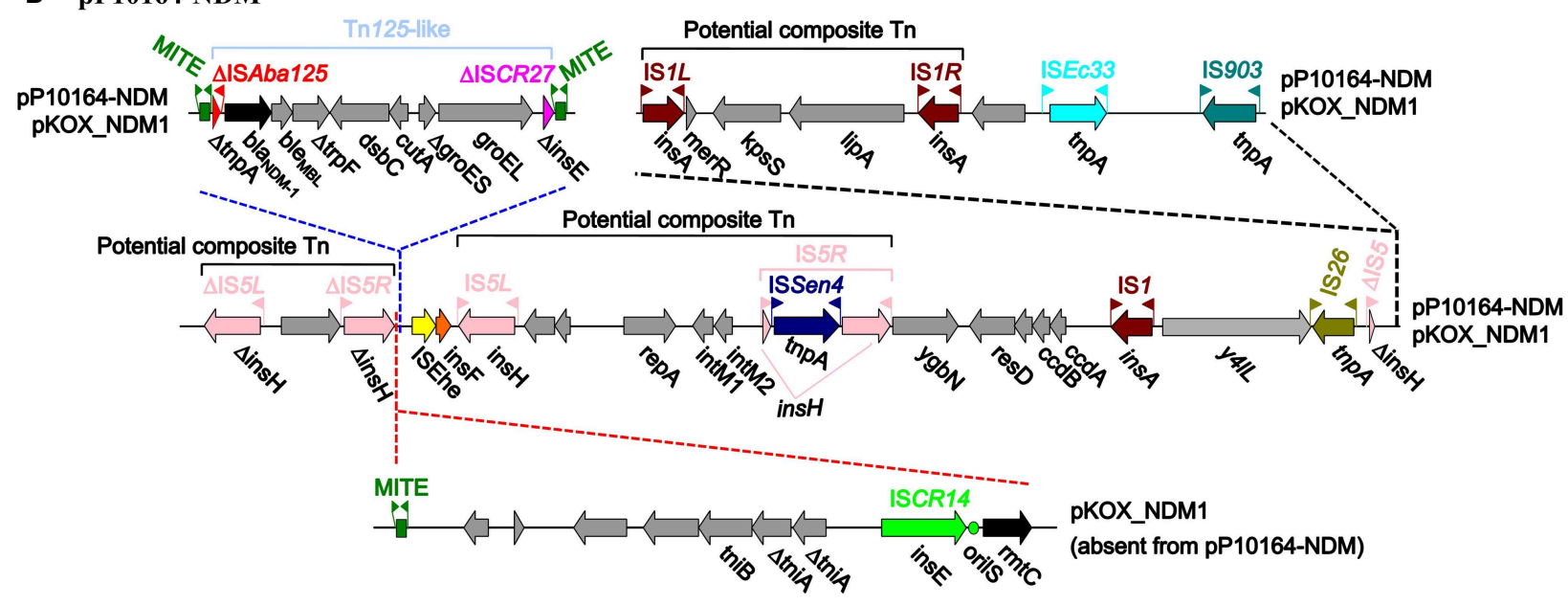

FIGURE 3 | Accessory modules of plasmids pYNKP001-NDM (A) and pP10164-NDM (B). Genes are denoted by arrows and colored based on gene function classification.

p271A (in E. coli from a patient following medical transfer from a hospital in Bangladesh to Australia) (Poirel et al., 2011).

The $b l a_{\mathrm{NDM}-1}$ genes of pYNKP001-NDM and pP10164-NDM are embedded in Tn125-like elements, which are characterized as two distinct truncated versions of the prototype NDM-encoding Tn125, as observed in pNDM-BJ01 (Hu et al., 2012).

The Tn125-like elements with a large array of derivatives represent the major genetic platforms for $b l a_{\mathrm{NDM}}$ genes across host bacteria (Bonnin et al., 2012; Mcgann et al., 2012; Partridge and Iredell, 2012; Zhang et al., 2013; Zong and Zhang, 2013; Fiett et al., 2014; Jones et al., 2014; Mataseje et al., 2014). Notably, flanking of Tn125-like elements by MITE or its remnant as observed herein indicates that MITE facilitates transposition and mobilization of $b l a_{\mathrm{NDM}-1}$ gene contexts.

\section{Acknowledgments}

This work was funded by the National Key Program for Infectious Disease of China (2013ZX10004216),
National High-Tech Research and Development Program (2014AA021402), and National Natural Science Foundation of China (31471184).

\section{Supplementary Material}

The Supplementary Material for this article can be found online at: http://journal.frontiersin.org/article/10.3389/fmicb. 2015.00458/abstract

Figure S1 | Detection of carbapenemase activity. In the presence of any carbapenemase, relevant carbapenems are hydrolyzed and transformed into its carboxylic form, leading to a $\mathrm{pH}$ decrease, which is detected by a color change of phenol red solution (red to yellow-orange). Ambler class A carbapenemases are at least partially inhibited by tazobactam, whereas class B carbapenemases (metallo- $B$-lactamases) are inhibited by divalent cation chelators, such as EDTA. There is no available chemical inhibitor for class D carbapenemases. In this study,

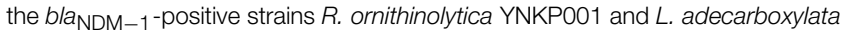
P10164, E. coli YNKP001-NDM-TB1 and P10164-NDM-EC600, and K. pneumoniae ATCC BAA-2146 (positive control) (Rasheed et al., 2013) exhibited class B carbapenemase activity. As expected, E. coli EC600 and DH10B had no carbapenemase activity. 


\section{References}

Bali, R., Sharma, P., Gupta, K., and Nagrath, S. (2013). Pharyngeal and peritonsillar abscess due to Leclercia adecarboxylata in an immunocompetant patient. J. Infect. Dev. Ctries. 7, 46-50. doi: 10.3855/jidc.2651

Biedenbach, D., Bouchillon, S., Hackel, M., Hoban, D., Kazmierczak, K., Hawser, S., et al. (2015). Dissemination of NDM Metallo-beta-lactamase genes among clinical isolates of enterobacteriaceae collected during the SMART global surveillance study from 2008 to 2012. Antimicrob. Agents Chemother. 59, 826-830. doi: 10.1128/AAC.03938-14

Bonnin, R. A., Poirel, L., Naas, T., Pirs, M., Seme, K., Schrenzel, J., et al. (2012). Dissemination of New Delhi metallo-beta-lactamase-1-producing Acinetobacter baumannii in Europe. Clin. Microbiol. Infect. 18, E362-E365. doi: 10.1111/j.1469-0691.2012.03928.x

Castanheira, M., Deshpande, L. M., Dipersio, J. R., Kang, J., Weinstein, M. P., and Jones, R. N. (2009). First descriptions of blaKPC in Raoultella spp. (R. planticola and R. ornithinolytica): report from the SENTRY Antimicrobial Surveillance Program. J. Clin. Microbiol. 47, 4129-4130. doi: 10.1128/JCM.01502-09

Chen, Y. T., Lin, A. C., Siu, L. K., and Koh, T. H. (2012). Sequence of closely related plasmids encoding bla(NDM-1) in two unrelated Klebsiella pneumoniae isolates in Singapore. PLOS ONE 7:e48737. doi: 10.1371/journal.pone.00 48737

Chun, S., Yun, J. W., Huh, H. J., and Lee, N. Y. (2015). Clinical characteristics of Raoultella ornithinolytica bacteremia. Infection 43, 59-64. doi: 10.1007/s15010014-0696-z

Delihas, N. (2011). Impact of small repeat sequences on bacterial genome evolution. Genome Biol. Evol. 3, 959-973. doi: 10.1093/gbe/evr077

De Mauri, A., Chiarinotti, D., Andreoni, S., Molinari, G. L., Conti, N., and De Leo, M. (2013). Leclercia adecarboxylata and catheter-related bacteraemia: review of the literature and outcome with regard to catheters and patients. J. Med. Microbiol. 62, 1620-1623. doi: 10.1099/jmm.0.059535-0

Domingues, S., Toleman, M. A., Nielsen, K. M., and Da Silva, G. J. (2013). Identical miniature inverted repeat transposable elements flank class 1 integrons in clinical isolates of Acinetobacter spp. J. Clin. Microbiol. 51, 2382-2384. doi: 10.1128/JCM.00692-13

Dortet, L., Poirel, L., and Nordmann, P. (2012). Rapid identification of carbapenemase types in Enterobacteriaceae and Pseudomonas spp. by using a biochemical test. Antimicrob. Agents Chemother. 56, 6437-6440. doi: 10.1128/AAC.01395-12

Dortet, L., Poirel, L., and Nordmann, P. (2014). Worldwide dissemination of the NDM-type carbapenemases in Gram-negative bacteria. Biomed. Res. Int. 2014:249856. doi: 10.1155/2014/249856

Fiett, J., Baraniak, A., Izdebski, R., Sitkiewicz, I., Zabicka, D., Meler, A., et al. (2014). The first NDM metallo-beta-lactamase-producing Enterobacteriaceae isolate in Poland: evolution of IncFII-type plasmids carrying the bla(NDM-1) gene. Antimicrob. Agents Chemother. 58, 1203-1207. doi: 10.1128/AAC.01197-13

Frank, J. A., Reich, C. I., Sharma, S., Weisbaum, J. S., Wilson, B. A., and Olsen, G. J. (2008). Critical evaluation of two primers commonly used for amplification of bacterial 16S rRNA genes. Appl. Environ. Microbiol. 74, 2461-2470. doi: 10.1128/AEM.02272-07

Garcia-Fulgueiras, V., Seija, V., Aguerrebere, P., Cordeiro, N., and Vignoli, R. (2014). First report of a clinical isolate of Leclercia adecarboxylata harbouring multiple resistance genes in Uruguay and review of the literature. J. Glob. Antimicrob. Resist. 2, 77-81. doi: 10.1016/j.jgar.2014.01.002

Geffen, Y., Adler, A., Paikin, S., Khabra, E., Gorenshtein, S., Aronov, R., et al. (2013). Detection of the plasmid-mediated KPC-2 carbapenem-hydrolysing enzyme in three unusual species of the Enterobacteriaceae family in Israel. J. Antimicrob. Chemother. 68, 719-720. doi: 10.1093/jac/dks443

Hadano, Y., Tsukahara, M., Ito, K., Suzuki, J., Kawamura, I., and Kurai, H. (2012). Raoultella ornithinolytica bacteremia in cancer patients: report of three cases. Intern. Med. 51, 3193-3195. doi: 10.2169/internalmedicine.51.8349

Haruki, Y., Hagiya, H., Sakuma, A., Murase, T., Sugiyama, T., and Kondo, S. (2014). Clinical characteristics of Raoultella ornithinolytica bacteremia: a case series and literature review. J. Infect. Chemother. 20, 589-591. doi: 10.1016/j.jiac.2014.05.005

Hess, B., Burchett, A., and Huntington, M. K. (2008). Leclercia adecarboxylata in an immunocompetent patient. J. Med. Microbiol. 57, 896-898. doi: 10.1099/jmm.0.47673-0
Hu, H., Hu, Y., Pan, Y., Liang, H., Wang, H., Wang, X., et al. (2012). Novel plasmid and its variant harboring both a bla(NDM-1) gene and type IV secretion system in clinical isolates of Acinetobacter lwoffii. Antimicrob. Agents Chemother. 56, 1698-1702. doi: 10.1128/AAC.06199-11

Huang, T. W., Wang, J. T., Lauderdale, T. L., Liao, T. L., Lai, J. F., Tan, M. C., et al. (2013). Complete sequences of two plasmids in a $b l a_{N D M-1}$-positive Klebsiella oxytoca isolate from Taiwan. Antimicrob. Agents Chemother. 57, 4072-4076. doi: 10.1128/AAC.02266-12

Isozumi, R., Yoshimatsu, K., Yamashiro, T., Hasebe, F., Nguyen, B. M., Ngo, T. C., et al. (2012). bla(NDM-1)-positive Klebsiella pneumoniae from environment, Vietnam. Emerg. Infect. Dis. 18, 1383-1385. doi: 10.3201/eid1808.111816

Johnson, A. P., and Woodford, N. (2013). Global spread of antibiotic resistance: the example of New Delhi metallo-beta-lactamase (NDM)-mediated carbapenem resistance. J. Med. Microbiol. 62, 499-513. doi: 10.1099/jmm.0.052555-0

Jones, L. S., Carvalho, M. J., Toleman, M. A., White, P. L., Connor, T. R., Mushtaq, A., et al. (2014). Characterisation of plasmids in extensively drug-resistant (XDR) Acinetobacter species from India and Pakistan. Antimicrob. Agents Chemother. 59, 923-929. doi: 10.1128/AAC.03242-14

Kanki, M., Yoda, T., Tsukamoto, T., and Shibata, T. (2002). Klebsiella pneumoniae produces no histamine: raoultella planticola and Raoultella ornithinolytica strains are histamine producers. Appl. Environ. Microbiol. 68, 3462-3466. doi: 10.1128/AEM.68.7.3462-3466.2002

Keren, Y., Keshet, D., Eidelman, M., Geffen, Y., Raz-Pasteur, A., and Hussein, K. (2014). Is Leclercia adecarboxylata a new and unfamiliar marine pathogen? J. Clin. Microbiol. 52, 1775-1776. doi: 10.1128/JCM.03239-13

Khajuria, A., Praharaj, A. K., Grover, N., and Kumar, M. (2013). First report of bla $a_{N D M-1}$ in Raoultella ornithinolytica. Antimicrob. Agents Chemother. 57, 1092-1093. doi: 10.1128/AAC.02147-12

Mataseje, L. F., Boyd, D. A., Lefebvre, B., Bryce, E., Embree, J., Gravel, D., et al. (2014). Complete sequences of a novel bla $a_{N D M-1}$-harbouring plasmid from Providencia rettgeri and an FII-type plasmid from Klebsiella pneumoniae identified in Canada. J. Antimicrob. Chemother. 69, 637-642. doi: $10.1093 / \mathrm{jac} / \mathrm{dkt} 445$

Mau, N., and Ross, L. A. (2010). Raoultella ornithinolytica bacteremia in an infant with visceral heterotaxy. Pediatr. Infect. Dis. J. 29, 477-478. doi: 10.1097/INF.0b013e3181ce9227

Mazzariol, A., Zuliani, J., Fontana, R., and Cornaglia, G. (2003). Isolation from blood culture of a Leclercia adecarboxylata strain producing an SHV-12 extended-spectrum beta-lactamase. J. Clin. Microbiol. 41, 1738-1739. doi: 10.1128/JCM.41.4.1738-1739.2003

Mcgann, P., Hang, J., Clifford, R. J., Yang, Y., Kwak, Y. I., Kuschner, R. A., et al. (2012). Complete sequence of a novel 178-kilobase plasmid carrying bla(NDM1) in a Providencia stuartii strain isolated in Afghanistan. Antimicrob. Agents Chemother. 56, 1673-1679. doi: 10.1128/AAC.05604-11

Michael, Z., Mcgann, P. T., Alao, O., Stevenson, L., Lesho, E., and Viscount, H. (2013). Isolation of Leclercia adecarboxylata from an infected war wound in an immune competent patient. Mil. Med. 178, e390-e393. doi: 10.7205/MILMEDD-12-00382

Morais, V. P., Daporta, M. T., Bao, A. F., Campello, M. G., and Andres, G. Q. (2009). Enteric fever-like syndrome caused by Raoultella ornithinolytica (Klebsiella ornithinolytica). J. Clin. Microbiol. 47, 868-869. doi: 10.1128/JCM.01709-08

Netikul, T., Sidjabat, H. E., Paterson, D. L., Kamolvit, W., Tantisiriwat, W., Steen, J. A., et al. (2014). Characterization of an IncN2-type $b l a_{N D M-(1)^{-}}$ carrying plasmid in Escherichia coli ST131 and Klebsiella pneumoniae ST11 and ST15 isolates in Thailand. J. Antimicrob. Chemother. 69, 3161-3163. doi: 10.1093/jac/dku275

Nordmann, P., Poirel, L., Walsh, T. R., and Livermore, D. M. (2011). The emerging NDM carbapenemases. Trends Microbiol. 19, 588-595. doi: 10.1016/j.tim.2011.09.005

Ou, W., Cui, L., Li, Y., Zheng, B., and Lv, Y. (2014). Epidemiological characteristics of $b l a_{N D M-1}$ in Enterobacteriaceae and the Acinetobacter calcoaceticusAcinetobacter baumannii complex in China from 2011 to 2012. PLoS ONE 9:e113852. doi: 10.1371/journal.pone.0113852

Papagiannitsis, C. C., Studentova, V., Hrabak, J., Kubele, J., Jindrak, V., and Zemlickova, H. (2013). Isolation from a nonclinical sample of Leclercia adecarboxylata producing a VIM-1 metallo-beta-lactamase. Antimicrob. Agents Chemother. 57, 2896-2897. doi: 10.1128/AAC.00052-13 
Partridge, S. R., and Iredell, J. R. (2012). Genetic contexts of bla $a_{N D M-1}$. Antimicrob. Agents Chemother. 56, 6065-6067. doi: 10.1128/AAC.00117-12

Partridge, S. R., Paulsen, I. T., and Iredell, J. R. (2012). pJIE137 carrying blaCTXM-62 is closely related to p271A carrying bla ${ }_{N D M-1}$. Antimicrob. Agents Chemother. 56, 2166-2168. doi: 10.1128/AAC.05796-11

Poirel, L., Bonnin, R. A., Boulanger, A., Schrenzel, J., Kaase, M., and Nordmann, P. (2012). Tn125-related acquisition of bla $a_{N D M}$-like genes in Acinetobacter baumannii. Antimicrob. Agents Chemother. 56, 1087-1089. doi: 10.1128/AAC.05620-11

Poirel, L., Bonnin, R. A., and Nordmann, P. (2011). Analysis of the resistome of a multidrug-resistant NDM-1-producing Escherichia coli strain by high-throughput genome sequencing. Antimicrob. Agents Chemother. 55, 4224-4229. doi: 10.1128/AAC.00165-11

Poirel, L., Carrer, A., Pitout, J. D., and Nordmann, P. (2009). Integron mobilization unit as a source of mobility of antibiotic resistance genes. Antimicrob. Agents Chemother. 53, 2492-2498. doi: 10.1128/AAC.00033-09

Rasheed, J. K., Kitchel, B., Zhu, W., Anderson, K. F., Clark, N. C., Ferraro, M. J., et al. (2013). New Delhi metallo-beta-lactamase-producing Enterobacteriaceae, United States. Emerg. Infect. Dis. 19, 870-878. doi: 10.3201/eid1906.121515

Shin, G. W., You, M. J., Lee, H. S., and Lee, C. S. (2012). Catheter-related bacteremia caused by multidrug-resistant Leclercia adecarboxylata in a patient with breast cancer. J. Clin. Microbiol. 50, 3129-3132. doi: 10.1128/JCM. 00948-12

Solak, Y., Gul, E. E., Atalay, H., Genc, N., and Tonbul, H. Z. (2011). A rare human infection of Raoultella ornithinolytica in a diabetic foot lesion. Ann. Saudi Med. 31, 93-94. doi: 10.4103/0256-4947.75794

Villa, L., Garcia-Fernandez, A., Fortini, D., and Carattoli, A. (2010). Replicon sequence typing of IncF plasmids carrying virulence and resistance determinants. J. Antimicrob. Chemother. 65, 2518-2529. doi: $10.1093 / \mathrm{jac} / \mathrm{dkq} 347$

Walckenaer, E., Poirel, L., Leflon-Guibout, V., Nordmann, P., and NicolasChanoine, M. H. (2004). Genetic and biochemical characterization of the chromosomal class A beta-lactamases of Raoultella (formerly Klebsiella) planticola and Raoultella ornithinolytica. Antimicrob. Agents Chemother. 48, 305-312. doi: 10.1128/AAC.48.1.305-312.2004
Walsh, T. R., Weeks, J., Livermore, D. M., and Toleman, M. A. (2011). Dissemination of NDM-1 positive bacteria in the New Delhi environment and its implications for human health: an environmental point prevalence study. Lancet. Infect. Dis. 11, 355-362. doi: 10.1016/S1473-3099(11)7 0059-7

Yao, Q., Zeng, Z., Hou, J., Deng, Y., He, L., Tian, W., et al. (2011). Dissemination of the $\mathrm{rmtB}$ gene carried on IncF and IncN plasmids among Enterobacteriaceae in a pig farm and its environment. J. Antimicrob. Chemother. 66, 2475-2479. doi: $10.1093 / \mathrm{jac} / \mathrm{dkr} 328$

Zhang, W. J., Lu, Z., Schwarz, S., Zhang, R. M., Wang, X. M., Si, W., et al. (2013). Complete sequence of the bla(NDM-1)-carrying plasmid pNDM-AB from Acinetobacter baumannii of food animal origin. J. Antimicrob. Chemother. 68, 1681-1682. doi: 10.1093/jac/dkt066

Zhou, G., Guo, S., Luo, Y., Ye, L., Song, Y., Sun, G., et al. (2014), NDM-1-producing strains, family Enterobacteriaceae, in hospital, Beijing, China. Emerg. Infect. Dis. 20, 340-342. doi: 10.3201/eid2002.1 21263

Zong, Z. (2014). The complex genetic context of blaPER-1 flanked by miniature inverted-repeat transposable elements in Acinetobacter johnsonii. PLoS ONE 9:e90046. doi: 10.1371/journal.pone.0090046

Zong, Z., and Zhang, X. (2013). blaNDM-1-carrying Acinetobacter johnsonii detected in hospital sewage. J. Antimicrob. Chemother. 68, 1007-1010. doi: 10.1093/jac/dks505

Conflict of Interest Statement: The authors declare that the research was conducted in the absence of any commercial or financial relationships that could be construed as a potential conflict of interest.

Copyright $\odot 2015$ Sun, Yin, Feng, Qiu, Zhang, Luo, Yang, Yang, Wang, Chen, Xia and Zhou. This is an open-access article distributed under the terms of the Creative Commons Attribution License (CC BY). The use, distribution or reproduction in other forums is permitted, provided the original author(s) or licensor are credited and that the original publication in this journal is cited, in accordance with accepted academic practice. No use, distribution or reproduction is permitted which does not comply with these terms. 